\title{
Ganglion cysts of the wrist: pathophysiology, clinical picture, and management
}

\author{
Warren Gude · Vincent Morelli
}

Published online: 26 August 2008

(C) Humana Press 2008

\begin{abstract}
This article reviews what is known about ganglion cyst formation, natural history (50\% of cysts will spontaneously resolve), diagnosis, and management of this common malady. Although the exact mechanism of cyst formation is unknown, most current theories hold that extraarticular mucin "droplets" coalesce to form the main body of the tumor. Only subsequently are the "cyst wall" and pedicle (connecting the cyst to a nearby synovial joint) formed. Treatment options include watchful waiting, nonoperative aspiration/injection, and surgical removal. Although treatment is often unnecessary, many patients seeking consultation desire some form of definitive treatment. Cyst aspiration/injection is fraught with a high incidence of recurrence. Surgery generally results in lower rates of recurrence, but a higher incidence of complications. All current treatment options are suboptimal.
\end{abstract}

Keywords Cyst - Ganglion - Wrist ganglion ·

Ganglion cyst

\section{Pathophysiology}

Ganglia are benign soft tissue tumors most commonly encountered in the wrist, but which may occur in any joint.

\section{W. Gude}

Sports and Emergency Medicine, 600 N Robbins Rd, Boise, ID 83702, USA

e-mail:wwgude@yahoo.com

\section{Morelli $(\square)$}

Family and Sports Medicine, Meharry/Vanderbilt Family

Medicine, 1005 DB Todd BLvd, Nashville,

TN 37208, USA

e-mail: morellivincent@yahoo.com
Sixty to seventy percent of ganglion cysts are found in the dorsal aspect of the wrist and communicate with the joint via a pedicle. This pedicle usually originates not only at the scapholunate ligament [1], but also may arise from a number of other sites over the dorsal aspect of the wrist capsule [2]. Thirteen to twenty percent of ganglia are found on the volar aspect of the wrist, arising via a pedicle from the radio scaphoid/scapholunate interval, scaphotrapezial joint, or the metacarpotrapezial joint, in that order of frequency [3]. Ganglia arising from a flexor tendon sheath in the hand account for approximately $10 \%$ of ganglia. Occurrence in other joints as well as intraosseus and intratendinous ganglia are much less common [4].

Microscopically, the pedicle contains a tortuous lumen, connecting the cyst to the underlying joint [5]. The presence of this connection is supported by the intraoperative and arthrographic findings of Angelides and by Andren and Eiken who demonstrated movement of intra-articular contrast from the radiocarpal joint into the ganglia in $44 \%$ of patients with a dorsal wrist ganglion and $85 \%$ of patients with a volar wrist ganglion. As contrast does not appear to travel from the cyst into the joint, a one-way valve mechanism has been postulated $[6,7]$. Such a one-way valve is thought to be formed by the number of small "micro-cysts" present in the tissue surrounding the pedicle. These "micro-cysts" communicate with the primary ganglion and are felt to be part of the tortuous pedicle lumen, connecting cyst to joint, and, in the process, creating the one-way valve mechanism [8].

Evaluation via electron microscopy demonstrates the wall of the ganglion to be composed of randomly oriented sheets of collagen arranged in loose layers, one on top of another. Rare cells are present in the collagen sheets and appear to be fully functional fibroblasts or mesynchymal cells. It should be emphasized that since no synovial lining 
exists in these structures, they cannot be classified as true cysts. Though there are focal areas of mucinous degeneration in the cyst wall, neither significant global degenerative changes, necrosis nor inflammatory changes within the pseudocyst or surrounding tissues have been demonstrated $[9,10]$.

Analysis of cystic fluid reveals that it is a gelatinous material containing mainly hyaluronic acid and lesser amounts of glucosamine, globulins, and albumen. This fluid is different biochemically and is much thicker than intra-articular synovial fluid. The origin of the fluid has not been fully elucidated, but it has been postulated to arise from one of three mechanisms. First, it may originate from within the joint, pumped into the cyst by motion of the wrist, second, from an extra-articular degenerative process resulting in cyst formation and subsequent communication to the joint, or finally it may originate from mesenchymal cells within the cell wall. It is possible that a combination of these mechanisms contribute.

Unfortunately, the origin of the ganglion itself remains as enigmatic as the origin of its fluid. Theories on cyst genesis have been difficult to prove and most are unable to account for all of the known features of the ganglion cyst. The concept that the cyst is a simple herniation of the joint capsule is difficult to support in light of the lack of synovial lining within the cyst itself. The theory that ganglion have an inflammatory etiology has been debunked by pathologic studies showing no pericystic inflammatory changes [9, 11].

Three other theories though, still warrant consideration. In the first, joint stress (acute or chronic) may lead to a rent in the joint capsule and allow leakage of synovial fluid into the peri-articular tissue. Subsequent reaction between this fluid and local tissue results in the creation of the gelatinous cystic fluid and the formation of the cyst wall.

In support of this "capsular rent" theory, some authors have postulated that pre-existing joint pathology (periscaphiod ligamentous injury, etc.) is the underlying cause of rent/cyst formation. Joint abnormalities are thought to lead to altered biomechanics, eventual weakening of the capsule, and finally leakage of fluid, and cyst formation. However, despite arthroscopic findings confirming the presence of intra-articular joint pathology in $50 \%$ of ganglion patients, no correlation between this pathology and postoperative cyst recurrence can be demonstrated. This leads some to conclude that intra-articular pathology is not the inciting event in the "rent" theory of ganglion formation.

Alternatively, joint stress may lead to mucioid degeneration of adjacent extra-articular connective tissue with subsequent fluid accumulation and eventual cyst formation. This theory holds that the cyst and pedicle form a direct connection to the joint only after the creation of the cystic.
Lastly, some believe that joint stress may stimulate mucin secretion by the mesenchymal cells detected by electron microscopy in the surrounding tissues. The final common pathway of all of these theories is the coalescence of small pools of mucin to form the main cyst. Production of the surrounding pseudocapsule is induced by an unknown mechanism, though possibly from compression of surrounding tissues [4, 12-14].

\section{Clinical picture}

On examination, wrist ganglion are usually 1-2 $\mathrm{cm}$ cystic structures, feeling much like a firm rubber ball that is well tethered in place by its attachment to the underlying joint capsule or tendon sheath. There is no associated warmth or erythema and the cyst readily transilluminates. The clinical presentation is usually adequate for diagnosis, and X-ray evaluation is rarely indicated (except in the case of "occult wrist ganglion" where MRI is needed to make a diagnosis).

Symptoms include aching in the wrist that may also radiate up the patient's arm, pain with activity or palpation of the mass, decreased range of motion and decrease grip strength. Volar ganglia may also cause paresthesias from compression of the ulnar or median nerves or their branches [4, 15].

The cause of pain is unknown but, in the case of dorsal ganglia, has been postulated to be from compression of the terminal branches of the posterior interroseus nerve [16]. There is some difference of opinion as to the frequency of pain. Thornburg reports that a painless mass is the most common presenting complaint, and Gang and Makhlouf support this noting that less than one third of patients involved in their study reported pain and that it was invariably mild. Zubowicz and other authors report that most of their patients did not have pain [17, 18]. On the other hand, various other studies report pain in 70-100\% of patients [3, 8, 19, 20]. In one study, $89 \%$ of patients reported pain but only $19 \%$ felt that it interfered with normal daily activities [2]. The most likely conclusion is that pain, even when present, is more likely to be annoying than debilitating.

\section{Management}

The management of ganglion cysts has weighed on the conscious of medicine for some time. According to Heister (1743), "The inspissated matter of a ganglion may often be happily dispersed by rubbing the tumor well each morning with fasting saliva and binding a plate of lead upon it for several weeks successively ... Others ... prefer a bullet that has killed some wild creature, especially a stag. 
Sometimes, indeed, a recent ganglion will speedily vanish ... by adding a repeated pressure with the thumb or a wooden mallet. If none of these means prove effectual ... they may be safely removed by incision provided you are careful to avoid the adjacent tendons and ligaments. But as for rubbing them with the hand of a dead man and the like ... I presume, my reader will excuse me from insisting on them" [2]. Although the methods of Heister have since been abandoned by the medical community, other scientifically unfounded treatment methods have taken their place. As could be expected, results have proven inconsistent and unreliable. For example, closed rupture with digital pressure or a book, hence the name 'Bible cyst,' demonstrates recurrence rates of 22-64\% [21], and "offends the sensibilities of the fastidious patient or physician" [16].

Our current (suboptimal) treatment of choice is aspiration-the mainstay of nonsurgical treatment. Studies have shown remarkably variable rates of success. Zubowicz reported $85 \%$ success with up to three aspirations. He does, note that failure was much more common with each subsequent aspiration, a fact confirmed by other studies, of which some report no benefit with repeat aspiration $[22,23]$. The impressive results of Zubowicz have been elusive in subsequent investigations, and, most studies, even with repeat aspirations, demonstrate a success rate of only $30-50 \%$. It should be noted that aspiration appears to be significantly more successful in ganglia of the flexor tendon sheath of the hand with success up to $60 \%$ or $70 \%$ of the time [24-26]. Additionally, some authors note the poorest success rates with aspirations of volar ganglia of the wrist and recommend against this procedure due to the risk of adjacent structures, including the radial artery and the palmar cutaneous branch of the median nerve $[4,12$, 23]. However, Wright et al. report that "conservative treatment (of volar ganglia) with aspiration is often recommended" and aspiration of volar ganglia has been included in a number of studies [23, 24, 27]. Aspiration should probably be based on physician experience and patient symptomatology. This may be a use for ultrasoundguided aspiration as has been advocated by some $[11,15]$.

To improve upon the success rate of simple aspiration, numerous adjunctive measures have been developed. Based on the mistaken theory that ganglia are inflammatory in origin, Becker, in 1953, introduced steroid injection after aspiration. Derbyshire, in 1966, reported a remarkable $86 \%$ success rate, but in a study of only 22 patients, some of whom were followed for only 2 months, and such lofty results have not been reproduced. In fact, success is really no better than aspiration alone [22] as would be expected since there is no evidence supporting inflammation as the cause of ganglia. As noted by Thornburg, "Injection of corticosteroids at the time of aspiration has not proved beneficial."
To obtain more complete drainage of the cyst's mucinous contents, instillation of hyaluranidase prior to aspiration has been advocated. It has also been purported that hyaluronidase makes the ganglion wall more permeable to steroids instilled after aspiration, but there was no elaboration on the mechanism by which hyaluronidase would affect the collagenous cyst wall [Paul and Sochart]. Otu reported a success rate of $95 \%$ in the initial study of this technique. Subsequent trials have demonstrated much less optimistic results [14, 17, 21]. In fact, results appear no better than simple aspiration alone, probably based on the fact that neither technique addresses the actual cause of the cyst.

Several authors have attempted splinting the wrist after aspiration (and surgery) to prevent wrist motion from pumping synovial fluid through the one-way valve mechanism and refilling the cyst. This also has proven no more effective than simple aspiration and in postoperative patients leads to prolonged joint stiffness and decreased range of motion [20, 24, 25]. Ironically, since the use of steroids remains fairly common, a number of techniques have been developed that are actually designed to increase inflammation within the ganglia to enhance scarring and thus close the potential space of the empty cyst and prevent recurrence. These include aspiration with multiple punctures of the wall, aspiration with injection of a sclerosant, and a thread technique in which two loops of silk suture are passed through the cyst and the ends left protruding for 3 weeks. This is to provide a path for drainage of re-accumulated ganglion fluid to drain and to enhance scar formation via the presence of a foreign body. Multiple punctures and simple aspiration yield similar results [24, 25, 28]. Injection of sclerosant has been used in numerous patients in the past, and McEvedy reported an $82 \%$ success rate. This procedure, however, fell out of favor after studies demonstrated a connection between the ganglion and the joint $[5,6]$. Mackie et al. reassessed this technique in 1985, but had a 93\% recurrence rate. Additionally, they demonstrated significant inflammatory injury when the sclerosant was injected into the tendons of chickens and joints of rats. Lack of success and potential side effects have led to a general abandonment of this approach [5, 29]. The thread technique, though successful in a single study, has not been widely adopted based on concerns of infection [4, 30]. It seems logical that efforts at enhancing inflammation and subsequent scarring within the ganglia would meet with limited success because the wall of the cyst is essentially acellular, and, thus, possesses limited potential to produce any mediators of inflammation.

A review of the history of the studies listed in Table 1 indicates a pattern of remarkable early success that is unable to be reproduced in subsequent investigations. 
Table 1 Review of studies

\begin{tabular}{|c|c|c|}
\hline Method & Recurrence rate $(\%)$ & Follow-up \\
\hline \multicolumn{3}{|l|}{ Aspiration } \\
\hline 1. Nield and Evans & 59 & 1 year \\
\hline 2. Varley & 67 (no improvement with multiple aspirations) & 4 months \\
\hline 3. Zubowicz & 15 (multiple aspirations) & 1 year \\
\hline 4. Stephen et al. & 69 & \\
\hline 5. Dias and Buch & 47 & 2 and 5 years \\
\hline \multirow[t]{2}{*}{ 6. Westbrook } & 49 & 6 weeks \\
\hline & Average 51 & \\
\hline \multicolumn{3}{|l|}{ Aspiration and steroid } \\
\hline 1. Derbyshire & 14 & 2 months -5 years \\
\hline 2. Paul and Sochart & 51 & \\
\hline 3. Wright et al. & 83 (even with repeat aspirations) & \\
\hline \multirow[t]{2}{*}{ 4. Breidahl and Adler } & 60 & \\
\hline & Average 52 & \\
\hline \multicolumn{3}{|l|}{ Aspiration with hyaluronidase } \\
\hline 1. Otu & 5 & 6 months \\
\hline 2. Paul and Sochart & 51 & 2 years \\
\hline 3. Nelson & 43 & $1-8$ years \\
\hline \multirow[t]{2}{*}{ 4. Jagert } & 77 & 1 year \\
\hline & Average 44 & \\
\hline \multicolumn{3}{|l|}{ Aspiration and sclerosant } \\
\hline 1. McEvedy & 18 & 13 years \\
\hline \multirow[t]{2}{*}{ 2. Mackie } & 93 & 3 months \\
\hline & Average 55 & \\
\hline \multicolumn{3}{|l|}{ Aspiration with splinting } \\
\hline 1. Korman et al. & 48 (same as nonimmobilized) & 1 year \\
\hline \multirow[t]{2}{*}{ 2. Richman et al. } & 60 (better than nonimmobilized control) & 22 months \\
\hline & Average 54 & \\
\hline \multicolumn{3}{|c|}{ Aspiration with multiple puncture } \\
\hline 1. Stephen et al. & 78 & \\
\hline 2. Richman et al. & 64 & 22 months \\
\hline \multirow[t]{2}{*}{ 3. Korman et al. } & 49 & 12 months \\
\hline & Average 64 & \\
\hline
\end{tabular}

Potential causes of this variability include the fact that many studies are small and may introduce statistical error. Follow-up is often incomplete, at times, no better than $70-80 \%$, and of variable length [22, 25, 27, 28, 31, 32]. This is significant since the natural history of the ganglion is one of spontaneous regression in up to half of patients (Table 2). Studies with an extended period of follow-up may incorrectly include cysts that naturally resolved as an indication of a successful intervention. On the other hand, follow-up that is too brief may not provide adequate time for recurrence. In short, results from nonsurgical treatment appear no better than expectant management and carries up to a 5\% complication rate [27] (It should be noted that these same difficulties with study design are common in investigations of surgical ganglion management) [1, 2, 23]. For example, follow-up was only $28 \%$ in one study, and, clearly, overall results might be quite different with inclusion of such a large number of unaccounted for patients [3].

Surgical excision remains the gold standard for treatment of ganglion cysts. Prior to the work of Angelides, postoperative recurrence rates were as high as $40 \%$, results that were rivaled by expectant management. Since the adoption of surgical techniques that include excision of the entire ganglion complex, including cyst, pedicle, and a cuff of the adjacent joint capsule, recurrence rates have improved significantly. Some authors claim recurrence rates for dorsal wrist ganglia as low as 1-5\% [1] and as low as $7 \%$ for volar wrist ganglia [4]. Generally, higherreported rates of recurrence are attributed to inadequate dissection in which the tortuous duct system located at the joint capsule is not fully excised. 
Table 2 Expectant management

\begin{tabular}{lll}
\hline Study & $\begin{array}{l}\text { Spontaneous } \\
\text { resolution }\end{array}$ & Follow-up \\
\hline 1. Westbrook et al. (2002) & $5 / 11(45 \%)$ & 4 months \\
2. McEvedy [7] & $10 / 21(47.6 \%)$ & 12 years \\
3. Carp and Stout (1928) & $7 / 12(58 \%)$ & 3 years \\
4. Rossen and Walker (1989) & $22 / 29(76 \%)$ & $51 / 2$ years \\
$\quad$ Involved only children) & $20 / 29(70 \%)$ & 2 years \\
$\begin{array}{l}\text { 5. Dias and Buch [27] } \\
\text { 6. Zachariae and }\end{array}$ & $20 / 38(53 \%)$ & 2 and 5 years \\
Vibe-Hansen (1973) & $40 / 101(40 \%)$ & 6 years \\
& & \\
\hline
\end{tabular}

Despite the remarkably low postoperative recurrence rates often reported, a review of the literature actually appears to demonstrate higher rates of recurrence. A significant number of patients included in studies of dorsal wrist ganglia are actually undergoing a second surgery after cyst recurrence. This may triple the number of patients reported to have had a recurrence at the conclusion of the investigation. Even if only a small percentage of patients experienced a recurrence during the study period, it does not mean that the patients undergoing a repeat operation did not have a recurrence. Although the authors of the article may have exceptional skill and an exceedingly low recurrence rate, it would appear that in the context of day-to-day patient care, it may not be possible for all surgeons to obtain the results published by highly experienced surgeons or from high volume specialty hand clinics. Greendyke notes in his discussion that both recurrences in his study occurred "early in the authors experience with these lesions." It is likely that patients will seek care from surgeons with similar levels of experience, thus making recurrence more common than what is reported in many studies.

As noted above, adding in the patients who are undergoing a second surgery with those who experience a recurrence while the study is ongoing, the average rate of recurrence jumps from $5.6 \%$ to $16 \%$. (This calculation is the same if you exclude Satko and Ganesh, as they only studied children, and Angelides, who might be excluded for several reasons. First, prior to this study, complete excision of the ganglion complex was not as commonly practiced and could negatively skew the calculations. Second, no other study has even approached this degree of success, with a phenomenal recurrence rate of only $0.6 \%$.) The question is not whether recurrence of $5 \%$ or $7 \%$ can be achieved, but rather, what recurrence rate actually occurs in the general surgical community. Although Angelides achieved remarkable success, it has not been demonstrated that these results will be consistently replicated.
The complication rate and recurrence rate of volar wrist ganglia are not the same as that of dorsal wrist ganglia. As the origin of volar ganglia is more variable and access is more difficult due to adjacent neurovascular structures, recurrence of volar ganglia may be as high as $42 \%$ and may average $30 \%$. Complications are also more common, reaching rates $>20 \%$ in some studies [20,27]. Jacobs and Govaers identified surgeon inexperience as a risk factor for higher recurrence rates and concluded "one cannot be confident that surgical excision of volar ganglia will be successful."

Surgery improves the rate of ganglion resolution and generally provides good to excellent results, but is not a panacea, and thus, the development and continued use of numerous nonsurgical techniques. It has been estimated that initial nonsurgical management of patients would save 100,000 dollars per 100 patients, though this study probably reports an unrealistically high success rate with aspiration (85\%) and assumes that all patients with a recurrence will opt for surgery [32]. Additionally, there is the cost of postoperative time lost from work, which is documented to be as long as 24 days and may average as long as 2 weeks. This may represent a significant indirect cost $[8,16,20,27,33]$. Despite excellent results by a number of authors with low complication and recurrence rates, surgery is not without risk. Complications include infection, neuroma, unsightly scar, and keloid. Additionally, postoperative stiffness, grip weakness, and decreased range of motion may occur. Rizzo reported stiffness in $25 \%$ of patients and required up to 8 weeks and occupational therapy to regain maximal function. Wright reported that $14 \%$ of patients complained of limitation of activities due to loss of wrist motion. Postoperative pain is not uncommon [8, 19, 32, 33]. Several authors have documented a relationship to open ganglionectomy and scapholunate instability. It has, though, been postulated that instability is not the result of surgery, but rather the pre-existing cause of the ganglion [13, 17, 34, 35]. Damage to the palmar cutaneous branch of the median nerve and radial artery are at risk during surgery for volar ganglia [4, 20,27]. Dias and Buch report a recurrence rate of $42 \%$ and a complication rate of $20 \%$ in their study of volar ganglia. Additionally, $8 \%$ of their patients report being worse postoperatively. Clay and Clement noted that $21 \%$ of their patients undergoing surgery of dorsal wrist ganglia were no better or worse after surgery. And this number does not include the fact that $11 \%$ of patients involved in the study were undergoing a repeat excision for a recurrent cyst.

Equally informative is the fact that many patients who have a postoperative or even postaspiration cyst recurrence elect to not undergo a second operation (Table 3). Wright et al. operated on only $29 \%$ of recurrences and Jacobs and Govaers insightfully noted, "Only five of twenty [patients] 
Table 3 (a) Patients undergoing procedures (aspiration, injection, etc.) who experience recurrence and request further intervention and (b) patients undergoing surgery who experience cyst recurrence and request further intervention

Further intervention (surgery)

\begin{tabular}{lc}
\hline (a) Procedure & \\
1. Stephen et al. & $21 / 119(18 \%)$ \\
2. Wright et al. & $12 / 24(50 \%)$ \\
3. Varley et al. & $22 / 57(38 \%)$ \\
4. Westbrook et al. & $2 / 50(4 \%)$ \\
(b) Surgery & \\
1. Wright et al. & $4 / 14(29 \%)$ \\
2. Jacobs and Govaers & $5 / 20(25 \%)$ \\
\hline
\end{tabular}

with recurrence had enough symptoms from it to warrant a second operation."

This is not to imply that surgery is not the most effective available treatment option or that complications are so onerous as to make surgery too risky. Rather it appears to represent the patient's perception of the ganglion as a disease. In the only study to address why patients actually seek medical attention for ganglia, Westbrook found that $38 \%$ expressed cosmetic concerns, $28 \%$ were concerned about malignancy, and only $26 \%$ presented because of pain. This supports the conclusions of other authors that pain, no matter how commonly it is present, is rarely significant or impairs activity and is not usually the impetus for seeking medical care $[2,7,17,18,20,23,30,32]$. As cosmesis is a common concern, surgery may simply be exchanging an unsightly lump with a $50 \%$ chance of spontaneous resolution for an unsightly and permanent scar [16, 20].

The literature supports the fact that a significant number of ganglia will undergo spontaneous resolution with some authors stating that, in the majority this will occur with in a couple of years (Table 2). An explanation of the benign nature of the cyst, combined with a discussion of its natural history of regression will often alleviate patient fears and desire for surgery. This may be especially true with aspiration of the cyst to provide temporary relief of any symptoms and to demonstrate the innocuous nature of its contents $[4,18]$. Westbrook noted that in $52 \%$ of patients treated via aspiration or with expectant management, the ganglion was still present at 6 weeks, yet none requested further intervention. Out of a total of 50 patients, only two consented to surgery. In numerous operative and nonoperative studies, patient satisfaction far exceeds the actual rate of ganglion resolution, implying that concerns over what the ganglion represents are more important to the patient than its actual presence [17, 18, 22, 27]. It would appear that the ganglion cyst is more commonly a nuisance than a significant medical problem, even in the mind of the patient. The cyst will often be tolerated in lieu of an invasive procedure [22, 28]. In the absence of function limiting symptoms, McEvedy may have been correct when he commented that ganglia are a benign condition "taking up the time of competent surgeons who might be more usefully engaged."

\section{Conclusion}

The interest in ganglia and the wide variety of treatments that have been developed are probably a result of its frequent presentation to physicians and lack of a fully satisfactory mode of treatment. Aspiration is a beneficial and simple option for acute management, but appears to have little long-term impact on resolution. Current adjunctive measures, on the other hand, provide no benefit over aspiration alone. Surgery offers improved resolution of ganglia, but is often more invasive than is warranted by patient symptoms [31]. Individual patient concerns regarding the ganglion cyst must first be ascertained. Using a thorough knowledge of the natural history of ganglia and the available treatment options and outcomes, the physician can provide guidance as the patient selects the most appropriate course of action for their circumstances.

\section{References}

1. Angelides AC, Wallace PF. The dorsal ganglion of the wrist: its pathogenesis, gross and microscopic anatomy, and surgical treatment. J Hand Surg. 1976;1(3):228-35.

2. Clay NR, Clement DA. The treatment of dorsal wrist ganglia by radical excision. J Hand Surg. 1988;13(2):187-91.

3. Greendyke SD, Wilson M, Shepler TR. Anterior wrist ganglia from the scaphotrapezial joint. J Hand Surg. 1992;17(3):487-90.

4. Thornburg LE. Ganglions of the hand and wrist. J Am Acad Orthop Surg. 1999;7(4):231-38.

5. Tophoj K, Henriques U. Ganglion of the wrist-a structure developed from the joint. Acta Orthop Scand. 1971;42(3): 244-50.

6. Andren L, Eiken O. Arthrographic studies of wrist ganglions. J Bone Joint Surg Am. 1971;53(2):299-302.

7. McEvedy BV. The simple ganglion. A review of modes of treatment and an explanation of the frequent failures of surgery. Lancet. 1954;266(6803):135-6.

8. Osterman AL, Raphael J. Arthroscopic treatment of dorsal ganglion of the wrist. Hand Clin. 1995;11:7-12.

9. Psalia JV, Mansel RE. The surface ultrastructure of ganglia. J Bone Joint Surg Br. 1978;60-B(2):228-33.

10. Loder RT, Robinson JH, Jackson WT, Allen DJ. A surface ultrastructure study of ganglia and digital mucous cysts. J Hand Surg. 1988;13(5):758-62.

11. Breidahl WH, Adler RS. Ultrasound-guided injection of ganglia with corticosteroids. Skeletal Radiol. 1996;25(7):635-8.

12. Nahra ME, Bucchieri JS. Ganglion cysts and other tumor related conditions of the hand and wrist. Hand Clin. 2004;20(3):249-60.

13. Duncan KH, Lewis RC. Scapholunate instability following ganglion cyst excision: a case report. Clin Orthop Relat Res. 1988;228:250-3. 
14. Jagers M, Akkerhuis P, Van Der Heijden M, Brink PRG. Hyaluronidase versus surgical excision of ganglia: a prospective, randomized clinical trial. J Hand Surg. 2002;27(3):256-8.

15. Nishikawa S, Toh S, Miura K, Arai K, Irie T. Arthroscopic diagnosis and treatment of dorsal wrist ganglion. J Hand Surg. 2001;26(6):547-9.

16. Derbyshire RC. Observations on the treatment of ganglia with a report on hydrocortisone. Am J Surg. 1966;112(5):635-6.

17. Paul AS, Sochart DH. Improving the results of ganglion aspiration by the use of hyaluronidase. J Hand Surg. 1997;22(2): 219-21.

18. Westbrook AP, Stephen AB, Oni J, Davis TR. Ganglia: the patient's perception. J Hand Surg. 2000;25(6):566-7.

19. Rizzo M, Berger R, Steinmann S, Bishop A. Arthroscopic resection in the management of dorsal wrist ganglions: results with a minimum two-year follow-up period. J Hand Surg. 2004;29(1):59-62.

20. Jacobs LGH, Govaers KHM. The volar wrist ganglion: just a simple cyst? J Hand Surg. 1990;15(3):342-6.

21. Nelson CL, Sawmiller S. Phalen GS: ganglions of the wrist and hand. J Bone Joint Surg Am. 1972;54(7):1459-64.

22. Varley GW, Neidoff M, Davis TRC, Clay NR. Conservative management of wrist ganglia: aspiration versus steroid infiltration. J Hand Surg. 1997;22(5):636-7.

23. Wright TW, Cooney WP, Ilstrup M. Anterior wrist ganglion. J Hand Surg. 1994;19(6):954-8.

24. Richman JA, Gelberman RH, Engber WD, Salamon PB, Bean DJ. Ganglions of the wrist and digits and results of treatment by aspiration and cyst wall puncture. J Hand Surg. 1987;12(6): 1041-3.

25. Korman J, Pearl R, Hentz VR. Efficacy of immobilization following aspiration of carpal and digital ganglions. J Hand Surg. 1992;17(6):1097-9.

26. Bruner JM. Treatment of "sesamoid" synovial ganglia of the hand by needle rupture. J Bone Joint Surg Am. 1963;45:1689-90.

27. Dias J, Buch K. Palmar wrist ganglion: does intervention improve outcome? J Hand Surg. 2003;28(2):172-6.

28. Stephen AB, Lyons AR, Davis TRC. A prospective study of two conservative treatments for ganglia of the wrist. J Hand Surg. 1999;24(1):104-5.

29. Mackie IG, Howard CB, Wilkens P. The dangers of sclerotherapy in the treatment of ganglia. J Hand Surg. 1984;9(2):181-4.

30. Gang RK, Makhlouf S. Treatment of ganglia by a thread technique. J Hand Surg. 1988;13(2):184-6.

31. Oni JA. Letter to the editor. J Hand Surg. 1993;18B:410.

32. Zubowicz VN, Ischii $\mathrm{CH}$. Management of ganglion cysts of the hand by simple aspiration. J Hand Surg. 1987;12(4):618-20.

33. Luchetti R, Badia A, Alfarano M, Orbay J, Indriago I, Mustapha B. Arthroscopic resection of dorsal wrist ganglia and treatment of recurrences. J Hand Surg. 2000;25(1):38-40.

34. Crawford GP, Taleisnik J. Rotary subluxation of the scaphoid after excision of dorsal carpal ganglion and wrist manipulation: a case report. J Hand Surg. 1983;8(6):921-5.

35. Watson HK, Rogers WD, Ashmead DIV. Re-evaluation of the cause of the wrist ganglion. J Hand Surg. 1989;14(5):812-7. 